\title{
Z-POLYNOMIALS AND RING COMMUTATIVITY
}

\author{
S.M. BUCKLEY AND D. MACHALE
}

\begin{abstract}
We characterise polynomials $f$ with integer coefficients such that a ring with unity $R$ is necessarily commutative if $f(x)$ is central for all $x \in R$. We also solve the corresponding problem without the assumption that the ring has a unity.
\end{abstract}

\section{INTRODUCTION}

In [4] and [1], characterisations were given for the polynomials $f$ with integer coefficients such that a ring $R$ is necessarily commutative whenever $f(x)=0$ for all $x \in R$. Here we characterise those polynomials $f$ such that a ring $R$ is necessarily commutative whenever $R$ satisfies the weaker condition that $f(x)$ is central for all $x \in R$. The fact that these two classes of polynomials are different follows from the observation that a ring satisfying the identity $x^{2}-2 x=0$ is necessarily commutative, while there are easy examples to show that there are non-commutative rings where $x^{2}-2 x$ is central for all $x$.

Throughout this paper, $f(X)=\sum_{i=1}^{n} a_{i} X^{i} \in X \mathbb{Z}[X]$. Given a ring $R$, we write $f(R)=0$ if $f(x)=0$ for all $x \in R$, and we write $f(R) \subset Z(R)$ if $f(x) \in Z(R)$ for all $x \in R$; here $Z(R)$ is the centre of $R$. For us, a ring does not necessarily have a unity, unless this is assumed.

Given a class $\mathcal{F}$ of rings, we denote by $C_{0}(\mathcal{F})$ and $C_{Z}(\mathcal{F})$ the sets of polynomials $f \in X \mathbb{Z}[X]$ that force a ring $R \in \mathcal{F}$ to be commutative whenever $f(x)$ always lies in $\{0\}$ or $Z(R)$, i.e.,

$$
\begin{aligned}
C_{0}(\mathcal{F}) & =\{f(X) \in X \mathbb{Z}[X]:(R \in \mathcal{F} \text { and } f(R)=0) \Longrightarrow R \text { commutative }\}, \\
C_{Z}(\mathcal{F}) & =\{f(X) \in X \mathbb{Z}[X]:(R \in \mathcal{F} \text { and } f(R) \subset Z(R)) \Longrightarrow R \text { commutative }\} .
\end{aligned}
$$

We are mainly interested in two classes $\mathcal{F}$ : the class of all rings $\mathcal{R}$, and the class of all rings with unity $\widetilde{\mathcal{R}}$. For each prime $p$, we also define the class $\mathcal{R}_{p}$ of rings such that $p^{k} R \subset Z(R)$ for some $k \in \mathbb{N}$, and the class $\widetilde{\mathcal{R}}_{p}:=\widetilde{\mathcal{R}} \cap \mathcal{R}_{p}$. We refer to polynomials in $C_{Z}(\mathcal{R})$ as Z-polynomials, and polynomials in $C_{0}(\mathcal{R})$ as C-polynomials.

A well-known result of Jacobson [3, Theorem 11] shows that for $n>1, X^{n}-X$ is a C-polynomial. More generally, Herstein [2] showed that if $a_{1}= \pm 1$, then $f$ is not just a C-polynomial, but also a Z-polynomial. In view of that result, we call $f$ a Herstein polynomial if $a_{1}= \pm 1$. 
Using Herstein's result, the second author and Laffey [4] showed that $f$ is a C-polynomial if and only if $f$ is either a Herstein polynomial, or $f$ satisfies the following set of three conditions: $a_{1}= \pm 2, a_{2}$ is odd, and $\sum_{i=2}^{n} a_{i}$ is odd. We will see that Z-polynomials form a more restrictive class than C-polynomials. In fact Z-polynomials coincide with Herstein polynomials; see Proposition 4.

Our characterisation of $C_{Z}(\widetilde{\mathcal{R}})$ is not as simple to state as that of $C_{Z}(\mathcal{R})$. It involves the following family of conditions indexed by a prime $p$ :

There is at least one non-multiple of $p$ among the numbers

$$
T_{p}:=\left\{a_{1}\right\} \cup\left\{b_{j} \mid 0 \leq j<p-1\right\},
$$

where

$$
b_{j}=\sum_{\substack{1 \leq i \leq n \\ i \equiv j(\bmod p-1)}} i a_{i}, \quad 0 \leq j<p-1 .
$$

Whenever the above condition holds, we say that $f$ satisfies the $T_{p}$ condition.

Theorem 1. Suppose $f(X)=\sum_{i=1}^{n} a_{i} X^{i} \in \mathbb{Z}[X]$. Then $f \in C_{Z}(\widetilde{\mathcal{R}})$ if and only if the greatest common divisor of the numbers $\left\{a_{i}\right\}_{i=1}^{n}$ is 1 , and $f$ satisfies the $T_{p}$ condition for all primes $p \leq n$ that divide $a_{1}$.

By comparison, we note that the main result in [1] states that a polynomial $f(X) \in X \mathbb{Z}[X]$ lies in $C_{0}(\widetilde{\mathcal{R}})$ if and only if the greatest common divisor of the numbers $\left\{a_{i}\right\}_{i=1}^{n}$ is 1 , and $f$ satisfies the $S_{p}$ condition for all primes $p \leq n / 2$ that divide $a_{1}$, where the $S_{p}$ condition involves a set $S_{p}$ is defined by:

$$
S_{p}:=T_{p} \cup\left\{c_{j} \mid 0 \leq j<p-1\right\},
$$

where

$$
c_{j}=\sum_{\substack{1 \leq i \leq n \\ i \equiv j(\bmod p-1)}} a_{i}, \quad 0 \leq j<p-1 .
$$

After reducing the problem to understanding $C_{Z}\left(\widetilde{\mathcal{R}}_{p}\right)$ for all primes $p$ in Section 2, we prove the main results in Section 3.

\section{REDUCTION TO PRIME POWERS}

There is one rather obvious necessary condition for $f \in C_{Z}\left(\widetilde{\mathcal{R}}_{p}\right)$ : given any prime $p$, the ring $G L_{2}\left(\mathbb{F}_{p}\right)$ is non-commutative and of characteristic $p$, so if every coefficient of $f$ is divisible by $p$ then $f \notin C_{0}\left(\widetilde{\mathcal{R}}_{p}\right) \supset C_{Z}\left(\widetilde{\mathcal{R}}_{p}\right)$. Thus every a polynomial in $C_{Z}(\widetilde{\mathcal{R}})$ (or in $\bigcap_{p \text { prime }} C_{Z}\left(\widetilde{\mathcal{R}}_{p}\right)$ ) is primitive, i.e. the greatest common divisor of its coefficients is 1 .

The rest of this section is dedicated to proving the following lemma which reduces the task of characterizing $C_{Z}(\widetilde{\mathcal{R}})$ to that of characterizing $C_{Z}\left(\widetilde{\mathcal{R}}_{p}\right)$ for all primes $p$. 
Lemma 2. $C_{Z}(\widetilde{\mathcal{R}})=\bigcap_{p \text { prime }} C_{Z}\left(\widetilde{\mathcal{R}}_{p}\right)$.

As a first step, the following simple lemma shows that commutativity of a ring $R$ such that $m R \subset Z(R)$ follows from commutativity of its subrings $R_{p}$ satisfying $p^{k} R_{p} \subset Z(R)$ for some $k \in \mathbb{N}$ and prime factor $p$ of $m$.

Lemma 3. Suppose $m R \subset Z(R)$, where $m \in \mathbb{N}$ has prime factorisation $m=\prod_{p \mid m} p^{k_{p}}$.
For each prime factor $p$ of $m$, let $m_{p}:=m / p^{k_{p}}$ and $R_{p}:=m_{p} R$. Then

(a) $R_{p}$ is an ideal in $R$, and $p^{k_{p}} R_{p} \subset Z(R)$.

(b) Every $x \in R$ can be written in the form

$$
x=z+\sum_{p \mid m} x_{p}, \quad z \in Z(R), x_{p} \in R_{p} .
$$

(c) $x y=y x$ whenever $x \in R_{p}, y \in R_{q}$, and $p, q$ are distinct prime factors of $m$.

(d) $R$ is commutative if and only if each $R_{p}$ is commutative.

Proof. Part (a) is immediate. As for (b), since the greatest common divisor of the numbers $\left\{m_{p}: p \mid n\right\}$ is 1 , we can choose $n_{p} \in \mathbb{Z}$ such that $\sum_{p \mid m} n_{p} m_{p}$ equals $1 \bmod m$, and then $x-\sum_{p} n_{p}\left(m_{p} x\right) \in Z(R)$.

We next prove (c). Let $x=m_{p} x^{\prime}, y=m_{q} y^{\prime}$. Since $m$ divides $m_{p} m_{q}$, we can use distributivity repeatedly to get

$$
x y=\left(\left(m_{p} m_{q}\right) x^{\prime}\right) y^{\prime}=y^{\prime}\left(\left(m_{p} m_{q}\right) x^{\prime}\right)=y x .
$$

Finally for (d), the "only if" part is trivial. Conversely, suppose that each of the rings $R_{p}$ is commutative. Given $x, y \in R$, we write

$$
x=z+\sum_{p \mid m} x_{p}, \quad y=w+\sum_{p \mid m} y_{p},
$$

where $z, w \in Z(R)$, and $x_{p}, y_{p} \in R_{p}$ for $p \mid m$. Using distributivity we expand $x y$ into a sum of products of pairs of elements from the set $\{z, w\} \cup\left(\cup_{p \mid m}\left\{x_{p}, y_{p}\right\}\right)$. Bearing in mind (c), we see that the factors in each of these products commute, and so $x y=y x$.

The degree $\operatorname{deg}(f)$ and codegree $\operatorname{codeg}(f)$ of a nonzero polynomial $f(X)=$ $\sum_{i=1}^{n} a_{i} X^{i}$ are the largest and smallest $i \in \mathbb{N}$, respectively, such that $a_{i} \neq 0$.

Proof of Lemma 2. Clearly $C_{Z}(\widetilde{\mathcal{R}}) \subset \bigcap_{p \text { prime }} C_{Z}\left(\widetilde{\mathcal{R}}_{p}\right)$, so we need only prove the reverse implication. Suppose therefore that $f \in \bigcap_{p \text { prime }} C_{Z}\left(\widetilde{\mathcal{R}}_{p}\right)$, so $f$ is necessarily primitive. Suppose also that $f(R) \subset Z(R)$ for some given unital ring $R$. $f$ must be of degree at least 1 . We write $f(X)=\sum_{i=1}^{n} a_{i} X^{i} \in \mathbb{Z}[X]$, where $a_{n} \neq 0$ and $n \in \mathbb{N}$, so $1 \leq \operatorname{codeg}(f) \leq \operatorname{deg}(f)=n$. 
If $\operatorname{codeg}(f)<\operatorname{deg}(f)$ then $g(X):=2^{n} f(X)-f(2 X)$ defines another nonzero polynomial such that $\operatorname{codeg}(g)=\operatorname{codeg}(f)$ and $\operatorname{deg}(g) \leq \operatorname{deg}(f)-1$. In fact

$$
g(X)=\sum_{i=1}^{n-1}\left(2^{n}-2^{i}\right) a_{i} X^{i}
$$

Also note that $g(R) \subset Z(R)$. Iterating this reduction procedure we eventually get a nonzero monomial such that $h(R) \subset Z(R)$. If $\operatorname{deg}(h)>1$, then simply replace $h$ by $H(X):=h(X+1)-h(1)$. Then $\operatorname{deg}(H)=\operatorname{deg}(h)$ and $\operatorname{codeg}(H)=1$, so if we again repeat the reduction procedure we eventually get a polynomial $F(X)=m X, m \in \mathbb{N}$, such that $F(R) \subset Z(R)$. Thus $m R \subset Z(R)$.

Define $m_{p}$ and $R_{p}$ as in Lemma 3 , and let

$$
R_{p}^{\prime}=\left\{m_{p} x+b \cdot 1 \mid x \in R, n \in \mathbb{Z}\right\} .
$$

Then for each prime factor $p$ of $m, R_{p}^{\prime}$ is a subring of $R, 1 \in R_{p}^{\prime}$, and $p^{k} R_{p}^{\prime} \subset$ $Z(R)$, so $R_{p}^{\prime} \in \widetilde{\mathcal{R}}_{p}$. Since also $f\left(R_{p}^{\prime}\right) \subset Z(R) \cap R_{p}^{\prime}=Z\left(R_{p}^{\prime}\right)$ for all $p$, and $f \in$ $C_{Z}\left(\widetilde{\mathcal{R}}_{p}\right)$, each $R_{p}^{\prime}$ is commutative. Thus also each $R_{p}$ is commutative, and so $R$ is commutative by Lemma 3 . But $R$ is an arbitrary ring satisfying $f(R) \subset Z(R)$, so we deduce that $f \in C_{Z}(\widetilde{\mathcal{R}})$, as required.

\section{Proofs of Results}

We first state and prove our characterisation of $C_{Z}(\mathcal{R})$.

Proposition 4. The classes of Z-polynomials and Herstein polynomials coincide.

Proof. The fact that Herstein polynomials are Z-polynomials is Herstein's main result in [2]. Conversely, as mentioned in the Introduction, it is shown in [4] that if $f(X)=\sum_{i=1}^{n} a_{i} X^{i} \in \mathbb{Z}[X]$ is a C-polynomial, then either it is a Herstein polynomial or $a_{1}= \pm 2$. Thus to establish our result, it suffices to exhibit a non-commutative ring $R$ such that $f(R) \subset Z(R)$ whenever $a_{1}$ is even.

This is rather easy to do: we simply take $(R,+, \cdot)$ to be the ring of $3 \times 3$ matrices over $\mathbb{Z}_{2}$ of the form

$$
\left(\begin{array}{lll}
0 & a & b \\
0 & 0 & c \\
0 & 0 & 0
\end{array}\right)
$$

This ring is not commutative since, for instance,

$$
\left(\begin{array}{lll}
0 & 1 & 0 \\
0 & 0 & 0 \\
0 & 0 & 0
\end{array}\right)\left(\begin{array}{lll}
0 & 0 & 0 \\
0 & 0 & 1 \\
0 & 0 & 0
\end{array}\right) \neq\left(\begin{array}{lll}
0 & 0 & 0 \\
0 & 0 & 1 \\
0 & 0 & 0
\end{array}\right)\left(\begin{array}{lll}
0 & 1 & 0 \\
0 & 0 & 0 \\
0 & 0 & 0
\end{array}\right) .
$$

However $2 x=x^{3}=0$ for all $x \in R$, Moreover since $x y z=0$ for all $x, y, z \in R$, it follows that $x^{2} \in Z(R)$ for all $x$. Thus if $a_{1}$ is even, then $f(x)=a_{2} x^{2} \in Z(R)$ for all $x \in Z(R)$. 
We now turn to the proof of Theorem 1. The main step is the following characterisation of $C_{Z}\left(\widetilde{\mathcal{R}}_{p}\right)$.

Theorem 5. Suppose $f(X)=\sum_{i=1}^{n} a_{i} X^{i} \in \mathbb{Z}[X]$, and let $p$ be a prime. Then $f \in C_{Z}\left(\widetilde{\mathcal{R}}_{p}\right)$ if and only if $f$ satisfies the $T_{p}$ condition.

Proof. We prove sufficiency of the $T_{p}$ condition. We may assume that $R \in \widetilde{\mathcal{R}}$ is such that $p^{k} R \subset Z(R)$ for some $k \in \mathbb{N}$. When considering $f(R) \subset Z(R)$ for such rings, we may treat the coefficients of $f$ as being either elements of $\mathbb{Z}_{p^{k}}$, or elements of $\mathbb{Z}$, as suits us.

If $p \nmid a_{1}$, then $a_{1}$ is a unit $\bmod p^{k}$, so $g(X):=a_{1}^{-1} f(X) \in \mathbb{Z}_{p^{k}}[X]$ has the form $X+\sum_{i=2}^{n} d_{i} X^{i}$, and so it is a Herstein polynomial when we view its coefficients as being integers. In particular the condition $g(R) \subset Z(R)$ forces characteristic $p^{k}$ rings $R \in \widetilde{\mathcal{R}}$ to be commutative. We may therefore assume that $p \mid a_{1}$.

Suppose that there exists $i, 0 \leq i<p-1$, such that $p \nmid b_{i}$. We treat $f(X)$ as a polynomial in $\mathbb{Z}_{p^{k}}[X]$, but let us also write $f_{p}(X)$ for $f(X)$ when instead viewed as an element of $\mathbb{Z}_{p}[X]$. Expanding $f_{p}(X+t)$ for $t \in \mathbb{Z}_{p}$, we see that the coefficient of $X$ is $s_{p}(t):=\sum_{i=1}^{n} i a_{i} t^{i-1}$. Let $S_{p}(X):=\sum_{i=0}^{p-1} b_{i} X^{i} \in \mathbb{Z}_{p}[X]$. By Fermat's Little Theorem, $s_{p}(t)=S_{p}(t)$ for all $t \in \mathbb{Z}_{p}$. The fact that $p \nmid b_{i}$ for some $i$ means that $S_{p}$ is not the zero polynomial, and so it has at most $p-1$ roots. Thus there exists $t \in \mathbb{Z}_{p}$ such that $s_{p}(t) \neq 0$. It follows that the coefficient of $X$ in the expansion of $f(X+t \cdot 1)$ is coprime to $p$ for some $t \in \mathbb{Z}_{p^{k}}$. Fixing this value of $t$ and picking $k \in \mathbb{Z}_{p^{k}}$ which is equivalent to $t \bmod p$, we get a polynomial $g(X):=f(X+k)-f(k) \in \mathbb{Z}_{p^{k}}[X]$ such that $g(R) \subset Z(R)$ and such that the coefficient of $X$ in $g$ is a unit $\bmod p^{k}$. This implies the commutativity of $R$ as before.

We now prove the converse. Suppose therefore that the $T_{p}$ condition fails for a given function $f$. Let $R$ be the ring of matrices

$$
x=\left(\begin{array}{lll}
\alpha & \beta & \delta \\
0 & \alpha & \gamma \\
0 & 0 & \alpha
\end{array}\right)
$$

where $\alpha, \beta, \gamma, \delta \in \mathbb{Z}_{p}$. For brevity, let us call $\alpha, \beta, \gamma, \delta$, the first, second, third, and fourth coordinates of $x$, respectively.

Given such a matrix $x$, it can be verified inductively that for all $i>1$,

$$
x^{i}=\left(\begin{array}{ccc}
\alpha^{i} & i \alpha^{i-1} \beta & * \\
0 & \alpha^{i} & i \alpha^{i-1} \gamma \\
0 & 0 & \alpha^{i}
\end{array}\right),
$$

where $*$ equals $i \alpha^{i-1} \delta+\left(\begin{array}{c}i \\ 2\end{array}\right) \alpha^{i-2} \beta \gamma$ (and $\alpha^{0}$ is defined to be 1 , even for $\alpha=0$ ), but the actual value does not affect subsequent calculations.

Consider now $f(x)$. Because $t^{p}=t$ for all $t \in \mathbb{Z}_{p}$, it follows from (1) that the second coordinate of $f(x)$ equals $a_{1} \beta+\sum_{i=0}^{p-2} d_{i} \alpha^{p+i-2} \beta$, where $d_{1}=b_{1}-a_{1}$ and 
$d_{i}=b_{i}$ for every other index in this sum, and the numbers $b_{i}$ are as in the $T_{p}$ condition. Now $T_{p}$ fails to hold, so $a_{i}$ and all the $b_{i}$ s are divisible by $p$, and so $p \mid d_{i}$ for $0 \leq i<p-1$. It follows that the second coordinate of $f(x)$ equals zero, and similarly we see that the fourth coordinate of $f(x)$ is 0 . Thus $f(x)$ has the form

$$
\left(\begin{array}{lll}
\varepsilon & 0 & \zeta \\
0 & \varepsilon & 0 \\
0 & 0 & \varepsilon
\end{array}\right)
$$

for some $\varepsilon, \zeta \in \mathbb{Z}_{p}$. But it is readily verified that all such matrices lie in the centre of $R$, so we have shown that $f(x) \in Z(R)$ for all $x \in R$ whenever $T_{p}$ fails. Now $R \in \widetilde{\mathcal{R}}_{p}$, and it is non-commutative regardless of $p$, since for instance

$$
\left(\begin{array}{lll}
0 & 1 & 0 \\
0 & 0 & 0 \\
0 & 0 & 0
\end{array}\right)\left(\begin{array}{lll}
0 & 0 & 0 \\
0 & 0 & 1 \\
0 & 0 & 0
\end{array}\right) \neq\left(\begin{array}{lll}
0 & 0 & 0 \\
0 & 0 & 1 \\
0 & 0 & 0
\end{array}\right)\left(\begin{array}{lll}
0 & 1 & 0 \\
0 & 0 & 0 \\
0 & 0 & 0
\end{array}\right) \text {. }
$$

Thus $f \notin C_{Z}\left(\widetilde{\mathcal{R}}_{p}\right)$ if the $T_{p}$ condition fails.

Proof of Theorem 1. Since

$$
C_{Z}(\widetilde{\mathcal{R}})=\bigcap_{p \text { prime }} C_{Z}\left(\widetilde{\mathcal{R}}_{p}\right)
$$

it follows that the polynomials in $C_{Z}(\widetilde{\mathcal{R}})$ are precisely those for which the $T_{p}$ condition holds for all primes $p$. If the gcd of the coefficients is not 1 , then all coefficients $a_{i}$ are divisible by some prime $p$, and certainly $f$ does not satisfy the $T_{p}$ condition. Thus by Theorem $5, f \notin C_{Z}(\widetilde{\mathcal{R}})$.

For the converse direction, since $T_{p}$ trivially holds when $p$ does not divide $a_{1}$, it suffices to show that the $T_{p}$ condition holds for all primes $p>n$ as long as the gcd of the coefficients is 1 . Because $p>n$, all the sums in the $T_{p}$ condition involve at most one term. Thus, since the gcd of the coefficients is 1 , there exists $i \leq n<p$ such that $p \nmid i a_{i}=b_{i}$.

The characterisation for quadratic polynomials is particularly simple, and follows immediately from Theorem 1.

Corollary 6. Suppose $f(X)=a_{1} X+a_{2} X^{2} \in \mathbb{Z}[X]$. Then $f \in C_{Z}(\widetilde{\mathcal{R}})$ if and only if $a_{1}$ is odd.

According to [4], a polynomial $f$ lies in $C_{Z}(\mathcal{R})$ if and only if it is a Herstein polynomial. Comparing this with Corollary 6 or Theorem 1 , it is easy to give examples of polynomials in $C_{Z}(\widetilde{\mathcal{R}}) \backslash C_{Z}(\mathcal{R})$, for instance $3 X+X^{2}$ or $5 X+2 X^{3}$. Comparing Theorem 1 with the characterisation of $C_{0}(\widetilde{\mathcal{R}})$ in [1], it is easy to give examples of polynomials in $C_{0}(\widetilde{\mathcal{R}}) \backslash C_{Z}(\widetilde{\mathcal{R}})$, for instance $3 X^{2}+2 X^{3}$ or $X^{2}$.

Lastly we note that the examples proving necessity in Theorem 5 (and so also in Theorem 1) involve only finite rings of prime characteristic. Thus if $\mathcal{F}$ is the 
set of all finite rings with unity, then $C_{Z}(\mathcal{F})=C_{Z}(\widetilde{\mathcal{R}})$, while if $\mathcal{F}$ consists of all finite rings with unity and characteristic $p$, then $C_{Z}(\mathcal{F})=C_{Z}\left(\widetilde{\mathcal{R}}_{p}\right)$. This is analogous to the fact that if $\mathcal{F}$ is the set of all finite rings (without the assumption of unity), then $C_{Z}(\mathcal{F})=C_{Z}(\mathcal{R})$ because the proof in [4] uses only finite rings to prove necessity.

\section{REFERENCES}

[1] S.M. Buckley and D. MacHale, Polynomials that force a unital ring to be commutative, Results Math., to appear; http://dx.doi.org/10.1007/s00025-012-0296-0.

[2] I.N. Herstein, The structure of a certain class of rings, Amer. J. Math. 75 (1953), 864-871; http://www.jstor.org/stable/2372554.

[3] N. Jacobson, Structure theory for algebraic algebras of bounded degree, Ann. Math. 46 (1945), 695-707; http://www.jstor.org/stable/1969205.

[4] T.J. Laffey and D. MacHale, Polynomials that force a ring to be commutative, Proc. Roy. Irish Acad. Sect. A 92A (1992), 277-280; http://www.jstor.org/stable/20489425.

S.M. Buckley:

Department of Mathematics and Statistics, National University of Ireland Maynooth, Maynooth, Co. Kildare, Ireland.

E-mail address: stephen.buckley@maths.nuim.ie

D. MacHale:

School of Mathematical Sciences, University College Cork, Cork, Ireland.

E-mail address: d.machale@ucc.ie 